\title{
Non-invasive control of influence of polyethylene glycol on transport function of fluorescent colored liposomal nanoparticles
}

O. Stelmashchuk, E. Zherebtsov, A. Zherebtsova, E. Kuznetsova, A. Vinokurov, et al.

O. Stelmashchuk, E. Zherebtsov, A. Zherebtsova, E. Kuznetsova, A. Vinokurov, A. Dunaev, A. Mamoshin, I. Snimshchikova, A. Borsukov, A. Bykov, I. Meglinski, "Non-invasive control of influence of polyethylene glycol on transport function of fluorescent colored liposomal nanoparticles," Proc. SPIE 10336, Saratov Fall Meeting 2016: Optical Technologies in Biophysics and Medicine XVIII, 1033604 (24 March 2017); doi: 10.1117/12.2269379 


\title{
Non-invasive control of influence of polyethylene glycol on transport function of fluorescent colored liposomal nanoparticles
}

\author{
O. Stelmashchuk ${ }^{\mathrm{a}^{*}}$, E. Zherebtsov ${ }^{\mathrm{a}, \mathrm{b}}$, A. Zherebtsova ${ }^{\mathrm{a}}$, E. Kuznetsova ${ }^{\mathrm{a}}$, A.Vinokurov $^{\mathrm{a}}$, A. Dunaev ${ }^{\mathrm{a}}$, \\ A. Mamoshin ${ }^{\mathrm{a}, \mathrm{c}}$, I. Snimshchikova ${ }^{\mathrm{a}}$, A. Borsukov ${ }^{\mathrm{c}}$, A. Bykov ${ }^{\mathrm{b}}$, I. Meglinski $^{\mathrm{b}}$ \\ ${ }^{a}$ Orel State University named after I.S. Turgenev, Komsomolskaya 95, Orel, Russia, 302026 \\ ${ }^{b}$ University of Oulu, Faculty of Information Technology and Electrical Engineering, Opto-Electronics and Measurement \\ Techniques, Erkki Koiso-Kanttilankatu 3, Oulu, Finland, 90570 \\ ${ }^{\mathrm{c}}$ Smolensk State Medical University, Problem Scientific and Research Laboratory "Diagnostic Researches and \\ Minimally invasive Technologies”, Krupskaya 40, Smolensk, Russia, 214019
}

\begin{abstract}
The studies were carried out on groups of clinically healthy mice line of outbred CD-1 stock. The model animals were divided into 2 groups and received experimental liposomal formulations. Using the method of fluorescence spectroscopy, we investigated the effectiveness of penetration into the circulatory system of fluorescently stained liposomes with polyethylene glycol (PEG) and without PEG when administered orally. Fluorescence channel with a fiber probe series of multifunctional laser non-invasive diagnostic system "LAKK-M" (SPE "LAZMA” Ltd, Russia) was used as the measuring equipment.
\end{abstract}

Keywords: liposomal nanoparticles, fluorescent label, polyethylene glycol, non-invasive control, fluorescence spectroscopy, transport function

*o.a.stelmashuk@gmail.com; phone 7919269 79 92; www.bmecenter.ru/en/fluorescence_spectroscopy_in_vivo

\section{INTRODUCTION}

In connection with the development of targeted drug delivery, monitoring the effectiveness of drug carriers penetration becomes necessary. Standard methods for monitoring medicament penetration during preclinical testing are usually carried out by invasive, time-consuming or expensive methods. Therefore, non-destructive optical methods become more frequently used in diagnosis ${ }^{1,2}$. Due to non-invasiveness, reliability, efficiency and quickness these methods based on principles of spectrophotometry and laser spectrum analysis, find applications in fields of medicine, pharmaceuticals, biology and chemistry ${ }^{3}$. Fluorescence spectroscopy can become the most promising optical diagnostic method for the control of medicines. This method is based on analyzing the characteristics of induced endogenous fluorescence in probed biological tissues with low-intensity optical radiation at certain wavelengths ${ }^{4,5}$. For in vivo measurements fluorescence spectrometers based on optical fiber systems are typically used ${ }^{6}$. Fluorescent labels are used to improve the reliability of results. Research for in vivo diagnostic purposes usually conducted using fluorescein and indocyanine green 7. Fluorescein has an excitation maximum at $494 \mathrm{~nm}$ and emission maximum at about $520 \mathrm{~nm}$. Fluorescein derivatives have a relatively high absorption capacity, a good quantum yield and water solubility.

Modern research of drug administration opens up a variety of methods, including targeting, based on using of micro- and nanoparticles, which protect drugs from negative action of environment like extreme $\mathrm{pH}$-values, enzyme-catalyzed degradation etc. Phospholipid liposomes are considered to be very effective in this aspect. And in case of other particles, their effectiveness is limited by relatively low possibility to overpass some physiological barriers such as mucosa and the cells of the intestinal epithelium. Increasing of liposomes penetrating capability may be achieved by the regulation and modification of their surface. Some researchers demonstrate using of polyethylene glycol (PEG) to increase the penetrating ability of nanoparticles ${ }^{8-10}$. Firstly, PEG was used for providing of prolonged circulation of nanoparticles in blood flow. And now it can be used for regulation of adhesion to intestinal mucosa and penetration of nanoparticles, but for the biological barriers. Than in this case the main characteristics of PEG is its molecular weight and density on the nanoparticle surface. For example, nanoparticles based on poly (methyl vinyl ether-co-maleic anhydride) with PEG-1000 $\left(\mathrm{M}_{\mathrm{w}} 1000 \mathrm{Da}\right)$ demonstrate higher adsorption capacity to the main component of mucosa - mucin. While the same nanoparticles with PEG-2000 $\left(\mathrm{M}_{\mathrm{w}} 2000 \mathrm{Da}\right)$ characterized by the higher diffusivity through mucosa ${ }^{11}$. The latter difference is much higher in the small intestine than in stomach. It's obvious that the effectiveness of drug administration 
by nanoparticles, including liposomes, will be determined by both these processes. The new methods of the research of such aspects are needed.

Fluorescent spectroscopy (FS) is becoming more widely used in chemistry, biology, in various fields of medical technology and medicine in general. The FS method is based on exciting fluorescence from tissue endogenous and exogenous fluorophores and recording the emission in the visible spectral region. This method is highly sensitive and provides a unique opportunity to study the excited states of molecules, photochemical reactions, dynamics of fast molecular processes, structures, and properties of complex biochemical and cellular systems ${ }^{12}$.

This paper demonstrates the perspectives of fluorescence spectroscopy method for the research of PEG influence on the liposome ability to transport drugs in blood at peroral administration. Tetrabromo fluorescein sodium salt (eosin-Y) was used as the fluorescent label. Methods for monitoring the penetration of drugs during preclinical testing were carried out by invasive methods in animal models conducted blood and tissues of interest ${ }^{13,14}$.

\section{THE METHOD OF RESEARCH}

Experimental studies were carried out on groups of clinically healthy mice outbred stock CD-1, formed by the analogue method. The model animals were divided into 2 groups which outside experiment received the same food. Three experimental groups received drugs by gavage for 4 weeks. The first group received liposomes without PEG $(\mathrm{n}=5)$. The second group received liposomes with PEG $(\mathrm{n}=5)$. Eosin concentration in the diet of group was $5 \mathrm{mg}$ per $\mathrm{kg}$ of body weight.

For this work liposomes were prepared using a modified version of the reverse-phase evaporation method ${ }^{15}$. Liposomes were prepared with and without addition of PEG. PEG was added at the stage of dissolution in chloroform. Phospholipids, cholesterol were dissolved in chloroform. The organic solvent was removed in a rotary evaporator under vacuum with the temperature of water bath $40{ }^{\circ} \mathrm{C}$ and rotor speed $100 \mathrm{rpm}$. Evaporation was stopped after thin lipid film formation. Ultrasound sonification was used for the hydration of lipid film. The duration of ultrasound treatment was chosen large enough for the complete film flushing and forming of liposomes with the diameter less than $500 \mathrm{~nm}$. Fluorescent dye eosin-Y (the disodium salt of 2,4,5,7 tetrabromofluorescein) was encapsulated inside the liposomes ${ }^{16-18}$. Passive inclusion was performed at the stage of the lipid film hydration. In case of empty liposomes lipid film was hydrated with the sterile saline. Unrelated eosin was removed from the liposome suspension using dialysis against saline.

Fluorescence spectrum of biological tissues was detected by means fluorescence channel with a fiber probe series of multifunctional laser non-invasive diagnostic system "LAKK-M" (SPE "LAZMA" Ltd, Russia), which was used as the measuring equipment (Fig. 1a). Measurements were performed on the proximal part of the tail (Fig.1b).

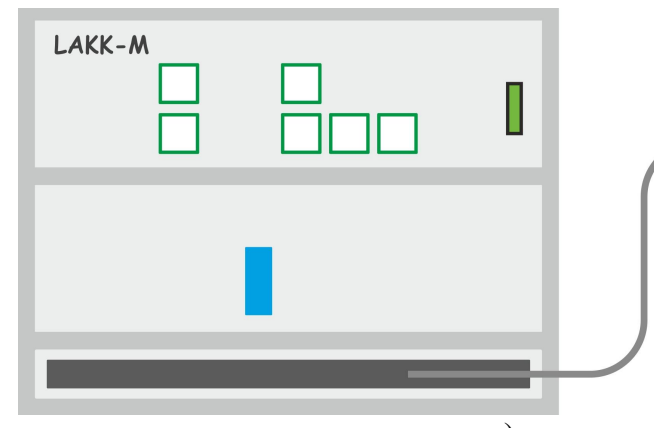

a)

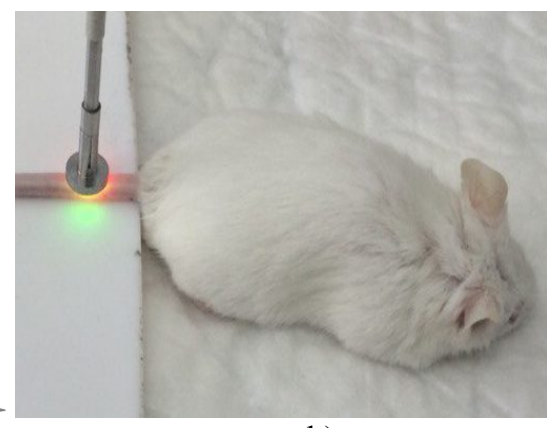

b)

Figure 1. Exterior view of the experimental setup (a) and the placement of an optical fiber to the proximal part of the tail (b).

Prior to administration of the drug out, the procedure of measuring the background fluorescence at two excitation wavelengths of $365 \mathrm{~nm}$ and $450 \mathrm{~nm}$ was carried (Fig.2). 


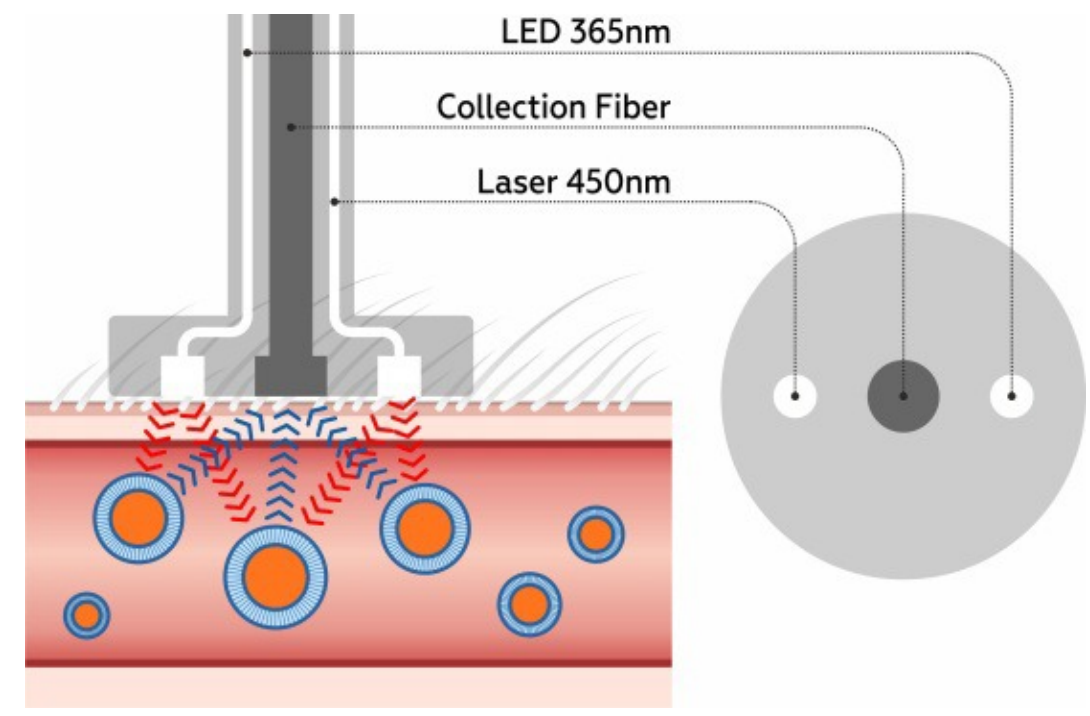

Figure 2. The fiber diagnostic probe used in the study

Due to its anatomical structure, the tail is well suited for fluorescence measurements bloodstream in upper layers of dermis. The tail has a well developed subcutaneous arterial and venous network with a large number of anastomoses between the vessels ${ }^{11}$. The area of skin under study was degreased with $96 \%$ ethanol solution before each measurement. Fluorescence spectra were recorded on the same wavelengths from the time of administration and further for 1,5 hours with an interval of $15 \mathrm{~min}$. Each measurement was carried out for a few seconds to reduce the effect of photo-bleaching, caused by the exhaustion of tissue fluorophores by irradiating light.

\section{RESULTS AND DISCUSSION}

Using in this work the method of fluorescence spectroscopy, we were able to evaluate the penetration effectiveness of liposomes with different chemical composition into the blood upon oral administration. Measurements at $365 \mathrm{~nm}$ exposure wavelength did not observe statistically significant data. This can be explained by low level penetration through the skin. The figures were obtained in the work when exposure at wavelength of $450 \mathrm{~nm}$ was shown. Results are expressed as group mean \pm standard deviation (in arbitrary units).

Liposomes without PEG included did not give a statistically significant changes in the fluorescence intensity (Fig. 3a). That may be associated with destruction of the particles under the aggressive environment influence of gastrointestinal tract and releasing the dye. Increasing of average peak fluorescence intensity from $240 \pm 39$ to $425 \pm 25$ a.u was observed in the group of mice which received liposome particles with PEG (Fig.3b).

Increased transportation of fluorescent label into the blood flow demonstrates the role of the used PEG-400 in liposomes penetration through mucosa and intestinal epithelium. On the one hand it makes liposomes-to-mucosa adhesion more intensive. This may be due to the reduced electrostatic repulsion in the system "liposomes-mucosa". Mucin fibers contain densely glycosylated and negatively charged regions. At the same time sunflower lecithin, which was used for liposome preparation, contains significant amount (comparing with phosphatidylcholine) of negatively charged phosphatidic acid and phosphatidylinositol ${ }^{19}$. So, liposomes without PEG may be repulsed by the mucosa. PEG layer masks the surface charge of nanoparticles. Besides, PEG may prevent nanoparticles aggregation by the formation of protective water layers. This makes liposomes little enough for the penetrating through dense porous structure of mucus gel. As a result the efficiency of liposome capture from intestinal lumen increased. On the other hand PEG molecular size was not enough for the strong binding of liposomes in the mucus. Due to the relatively low steric interaction and hydrophobic forces with the hydrophobic domains of mucin molecules, PEGylated liposomes characterized by the higher diffusivity through mucosa and ability of interaction with intestinal epithelium cells. 


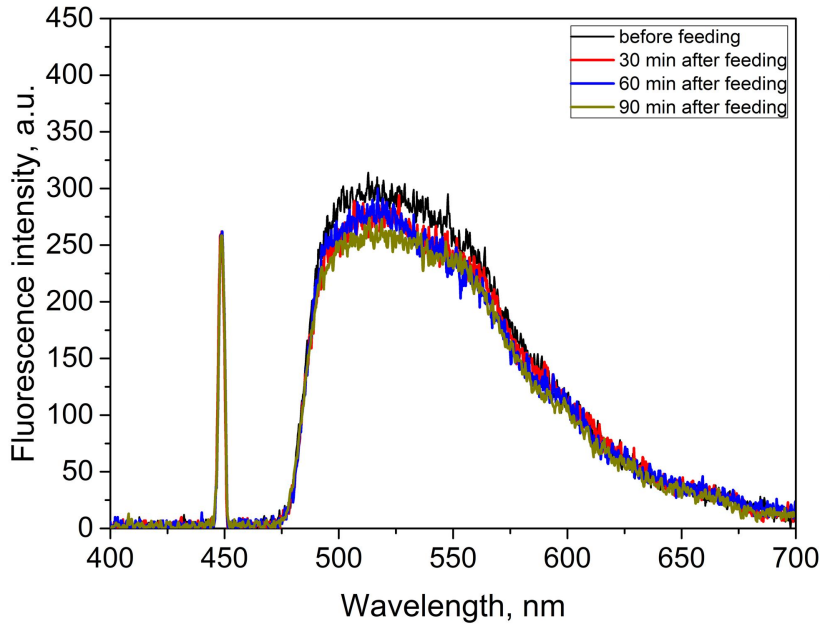

a)

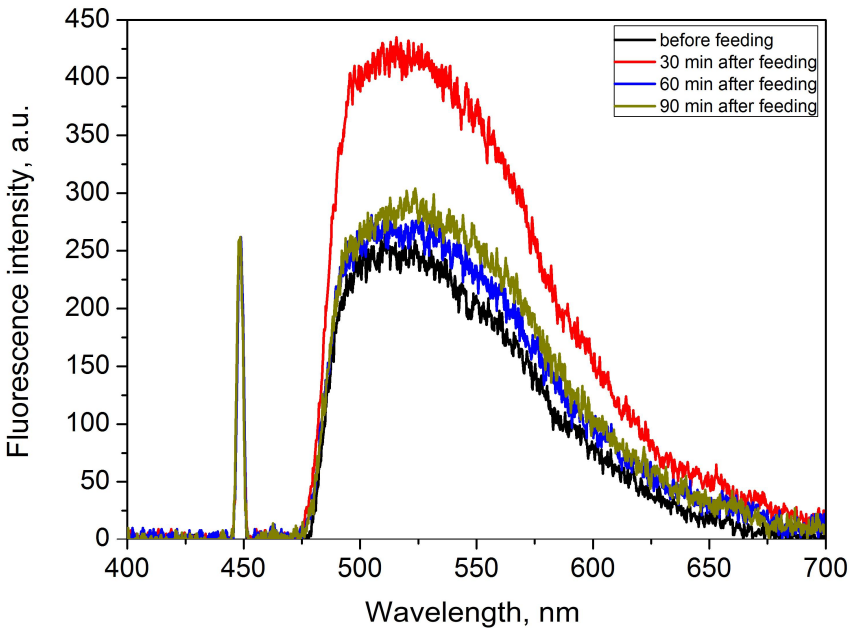

b)

Figure 3. Fluorescence spectra of the group received liposome without PEG (a) and group received liposome with PEG (b)

Using liposomes, thus more of fluorescent dye penetrates into the blood flow through the intestinal mucosa and gives a specific increase in fluorescence spectra. Maximum of intrinsic fluorescence of tested fluorescent dye (eosin-Y) lies in the region of $520 \pm 5 \mathrm{~nm}$. The maximum fluorescent response comes in the 30th minute after feeding. This can be explained by the time of passage through the gastrointestinal tract.

\section{CONCLUSION}

Modern laser and optical diagnostic methods can facilitate stages of research and development of new drugs. Applied for in vivo researches fluorescence spectroscopy allows to register the changes in the fluorescence spectra. These changes may occur as results of biological and functional changes and also under the influence of drugs. Fluorescent labels are often used for tracking drug forms in vivo. In this work, using fluorescent labels and fluorescence spectroscopy method, we were able to assess the transport functions of liposome particles administered orally. The results of this study can be used in the development of new drugs and high-throughput screening during their testing as well as theranostics. The experimental results suggest that the prepared multilayer liposome particles can increase the efficiency of the transported material passing into the blood flow from the gastrointestinal tract upon oral administration.

\section{ACKNOWLEDGMENTS}

The work was supported by grant of the President of the Russian Federation for state support of young Russian scientists № MK-7168.2016.8 and by the state task of the Ministry of Education and Science, Russian Federation, for the Orel State University named after I.S. Turgenev. Authors acknowledge the support of the Academy of Finland (grants: 296408 and 290596).

\section{REFERENCES}

[1] Kamali, T., Doronin, A., Rattanapak, T., Hook, S. and Meglinski, I., "Assessment of transcutaneous vaccine delivery by optical coherence tomography," Laser Physics Letters, 9(8), 607 (2012).

[2] Rattanapak, T., Birchall, J., Young, K., Ishii, M., Meglinski, I., Rades, T. and Hook, S., "Transcutaneous immunization using microneedles and cubosomes: Mechanistic investigations using Optical Coherence Tomography and Two-Photon Microscopy," J Control Release, 172(3), 894-903 (2013).

[3] Cooper, M. A., "Optical biosensors in drug discovery," Nat Rev Drug Discov, 1(7), 515-528 (2002). 
[4] Jacobi, U., Waibler, E., Bartoll, J., Schulze, P., Sterry, W. and Lademann, J., "In vivo determination of doxorubicin and its metabolites within the skin using laser scanning microscopy," Laser Physics Letters, 1(2), 100-103 (2004).

[5] Mycek, M. A. and Pogue, B. W., [Handbook of Biomedical Fluorescence], Taylor \& Francis, (2003).

[6] Utzinger, U. and Richards-Kortum, R. R., "Fiber optic probes for biomedical optical spectroscopy," J Biomed Opt, 8(1), 121-47 (2003).

[7] Brancato, R. and Trabucchi, G., "Fluorescein and indocyanine green angiography in vascular chorioretinal diseases," Semin Ophthalmol, 13(4), 189-98 (1998).

[8] Behrens, I., Pena, A. I., Alonso, M. J. and Kissel, T., "Comparative uptake studies of bioadhesive and nonbioadhesive nanoparticles in human intestinal cell lines and rats: the effect of mucus on particle adsorption and transport," Pharm Res, 19(8), 1185-93 (2002).

[9] Yoncheva, K., Lizarraga, E. and Irache, J. M., "Pegylated nanoparticles based on poly(methyl vinyl ether-comaleic anhydride): preparation and evaluation of their bioadhesive properties," Eur J Pharm Sci, 24(5), 411-9 (2005).

[10] Xu, Q., Ensign, L. M., Boylan, N. J., Schon, A., Gong, X., Yang, J. C., Lamb, N. W., Cai, S., Yu, T., Freire, E. and Hanes, J., "Impact of Surface Polyethylene Glycol (PEG) Density on Biodegradable Nanoparticle Transport in Mucus ex Vivo and Distribution in Vivo," ACS Nano, 9(9), 9217-27 (2015).

[11] Vanhoutte, G., Verhoye, M., Raman, E., Roberts, M. and Linden, A. V. d., "In-vivo non-invasive study of the thermoregulatory function of the blood vessels in the rat tail using magnetic resonance angiography," NMR in Biomedicine, 15(4), 263-269 (2002).

[12] Dunaev, A. V., Dremin, V. V., Zherebtsov, E. A., Rafailov, I. E., Litvinova, K. S., Palmer, S. G., Stewart, N. A., Sokolovski, S. G. and Rafailov, E. U., "Individual variability analysis of fluorescence parameters measured in skin with different levels of nutritive blood flow," Med Eng Phys, 37(6), 574-83 (2015).

[13] Huang, Y. Z., Gao, J. Q., Liang, W. Q. and Nakagawa, S., "Preparation and characterization of liposomes encapsulating chitosan nanoparticles," Biol Pharm Bull, 28(2), 387-90 (2005).

[14] Sercombe, L., Veerati, T., Moheimani, F., Wu, S. Y., Sood, A. K. and Hua, S., "Advances and challenges of liposome assisted drug delivery," Frontiers in Pharmacology, 6(DEC), (2015).

[15] Alves, A. C., Ramaldes, G. A., Oliveira, M. C., Cardoso, V. N., Mota-Santos, T. A., Faria, A. M. and Gontijo, C. M., "Ovalbumin encapsulation into liposomes results in distinct degrees of oral immunization in mice," Cell Immunol, 254(1), 63-73 (2008).

[16] Yefimova, S. L., Kurilchenko, I. Y., Tkacheva, T. N., Rozhkov, V. A., Sorokin, A. V., Lukianova, N. Y., Bezdenezhnykh, N. A., Malyukin, Y. V. and Chekhun, V. F., "Comparative study of dye-loaded liposome accumulation in sensitive and resistant human breast cancer cells," Exp Oncol, 34(2), 101-6 (2012).

[17] van Rooijen, N. and van Nieuwmegen, R., "Fluorochrome staining of multilamellar liposomes," Stain Technol, 53(6), 307-10 (1978).

[18] Chen, C.-S., Yao, J. and Durst, R. A., "Liposome encapsulation of fluorescent nanoparticles: Quantum dots and silica nanoparticles," Journal of Nanoparticle Research, 8(6), 1033-1038 (2006).

[19] Shulga, S., "Liposome stability dependence on fatty acid lecithin composition and sunflower phospholipids," Biotechnologia Acta, 9(1), 10 (2016). 